challenge for workers' personal-family life. The objective was to evaluate relationship of psychosocial factors and work-family interactions with psychological distress in Ecuadorian petroleum workers living transiently in camps, comparing to those who stay in cities.

Methods 350 workers invited to answer a self-administered questionnaire (response 88\%; October 2015 to January 2016). Psychological distress assessed through General Health Questionnare - 12 items (GHQ-12; dichotomized GHQ-score, $\mathrm{GHQ}_{\text {case }}=$ cut off 4/5). Psychosocial factors: Effort-Reward Imbalance (ERI) Questionnaire, short version (ERI and Overcommitment (OC) sub-scales, cut-off upper tertile). Work and family-personal life interaction evaluated with Work-Home Interaction - Nijmegen questionnaire (SWING; 4 dimensions, scores rescaled to $0-3$, cut-off upper tertile). Other variables were socio-demographics and commuting time from house or camp to workplace.

Result Higher prevalences of $\mathrm{GHQ}_{\text {cases }}$ were found in workers who perceived higher ERI $(38.1 \%$ vs $18.2 \%, \mathrm{p}<0.001)$ and OC levels $(33.6 \%$ vs $20.0 \%, \mathrm{p}<0.05)$, compared to lower tertiles). $\mathrm{GHQ}_{\text {cases }}$ were also more prevalent in urban workers (31.5\%, p=0.001) and when commuting time was $>15$ min $(31.5 \%, p<0.05)$. Negative interactions from work to family and viceversa were associated with higher prevalences of $\mathrm{GHQ}_{\text {cases }}(\mathrm{p}<0.05)$, while positive interactions were associated with lower ones $(\mathrm{p}<0.001)$. Adjusted Odds Ratios $(95 \% \mathrm{CI})$ for $\mathrm{GHQ}_{\text {case }}$ showed significant associations with ERI [2.34; (1.13-4.84)] and residing in cities while working [3.36; (1.388.16)]. Positive influences from domestic-personal life to work were associated with lower risk of psychological distress $[0.25$ (0.10-0.61)].

Discussion Perception of imbalance between effort and reward is associated with lower mental health. If skills and learnings acquired in domestic life are allowed to be displayed in work, workers seem to experience better mental health. Workers living transiently in camps exhibited better mental health in this group.

\section{PREVALENCE OF DEPRESSIVE SYMPTOMS ASSOCIATED TO NON-CONVENTIONAL WORK SCHEDULES AMONG ECUADORIAN INDUSTRIAL AND ARTISANAL FISHERMEN}

${ }^{1}$ Manuel Parra*, ${ }^{2}$ Mario Luna, ${ }^{1}$ Katja Radon, ${ }^{3}$ Denisse Carvalho. ${ }^{1}$ Center for International Health, University Hospital Munich (LMU). Munich- Germany; ${ }^{2}$ Tigre SA, Quito, Ecuador; ${ }^{3}$ Deparment of Community Heath, Universidad Federal do Paraná, Curitiba, Brasil

\subsection{6/oemed-2018-ICOHabstracts. 1624}

Introduction Workers' depression is an increasing concern for occupational health. Latin American researches have evaluated workplace psychosocial. Less is known about associations with working-hours schedules. This research was aimed to determine the prevalence of depressive symptoms associated with unconventional job schedules in industrial and artisanal fishermen in Ecuador.

Materials and methods 111 industrial (response 100\%) and 122 artisanal fishermen (response 80\%) were invited to answer a questionnaire, applied by a trained interviewer, in Manabí, Ecuador. Depressive symptoms in previous two weeks were evaluated with a validated version of Patients Health Questionnaire-9 items (PHQ9) each item scoring 0-3, total scale score from 0 to 27 ; cut-off $=5$. Job schedules were evaluated through a tailored set of questions including shifts, night work, hours per week, time of stay in the sea and rest period while in the sea. Other variables considered were socio-demographics (marital status, number of children, educational level). Adjusted logistic regression models were calculated to estimate Odds Ratios.

Results Both groups were similar in educational level and number of children. Industrial fishermen were older and lived more in couples, compared to artisanal ones. Industrial also had less employment security, they worked longer shifts and they had less rest periods in the sea. Artisanal had less formal job contracts. Depression was more prevalent in industrial fishermen $(36.0 \%$ vs $19.7 \%, \mathrm{p}<0.001)$. Longer time of stay in the sea showed a non-significant association with depression in both groups (OR 1.56 [0.61-4.04] for stays from 1 to 60 days, and 1.58 [0.52-4.72] for stays longer than 60 days). Adjusted model showed a significant association of depression with lack of rest periods in the sea (OR 11.5 [2.3-57.4]).

Discussion Depressive symptoms may increase in workers who have unconventional work schedules and without rest periods during the shift. Fishermen are exposed to such working conditions, mainly in the industrial sub-sector.

\section{INTRODUCTION OF A FATIGUE RISK MANAGEMENT SYSTEM IN A PAEDIATRIC HOSPITAL IN IRELAND}

${ }^{1}$ ME McMahon*, ${ }^{2} S$ Carolan. ${ }^{1}$ Children's University Hospital, Temple Street, Dublin 1, Ireland; ${ }^{2}$ Workplace Health and Wellbeing Unit, Health Services Executive, Adelaide Road, Dublin 2, Ireland

\subsection{6/oemed-2018-ICOHabstracts. 1625}

Introduction Fatigue is a common by-product of the 24 hour delivery of patient care. Defined as a decreased capacity to perform mental or physical work or the subjective state in which one can no longer perform a task, fatigue manifests in physiological performance decreases and cognitive impairment. $^{1}$ Fatigue thus poses elevated risk to the staff and patients of TSCUH and hospitals in Ireland and across the globe.

Methods The authors set out to introduce a Fatigue Risk Management System ${ }^{2}$ (FRMS) in a paediatric Hospital which would integrates management practices, beliefs and procedures to manage the risks associated with fatigue at the hospital. A review of the literature was conducted and a number of tools were utilised in undertaking the project including stakeholder analysis, risk assessment and a communication plan. The project was managed with reference to The HSE Change model. A local working group was formed to proactively manage this risk of fatigue with defined governance structures. The FRMS provides tailored defences against fatigue-related risks through the use of objective thresholds specifically for local environments. A policy was developed to support fatigue risk management system. Once the FRMS had been introduced locally a plan was devised to disseminate best practice at a national level.

Results A local working group has been established to address fatigue mitigation. Fatigue is now on the quality and risk agenda. Control strategies have been identified including: 
- the introduction of strategic napping

- purchase of flat bed chairs to facilitate same

- environmental - a dedicated nap station 'Snoozzone' proposed

- education programme on fatigue \& fatigue mitigation strategies introduced

- Fatigue risk scan is being completed and data collection is taking place

The fatigue scan is expected to influence the organisation of work and work scheduling to minimise the impact of fatigue and to provide adequate rest periods. The final FRMS (Fatigue Risk Management Strategy) document has now been completion.

Discussion Recent research shows that one in four doctors report driving home following work shifts when fatigued. There have been a number of deaths of doctors involved in road traffic accidents following night shifts in the UK in recent years associated with fatigue. Following the very positive feedback from staff following the successful introduction of a FRMS in a children's hospital, a programme to introduce FRMS is now being extended to public hospitals throughout Ireland. This will not only help to improve the health and safety of doctors and other healthcare workers but shall ultimately improve patient care. The FRMS shall be launched nationally later in 2017 in liaison with WHWU (Workplace Health and Wellbeing Unit), HSE to address fatigue in public funded hospitals in Ireland. A position paper is currently being written by the authors for Faculty of Occupational Medicine Ireland.

\section{EPIDEMIOLOGY IN THE WAITING ROOM: CAN THE OCCUPATIONAL PHYSICIAN DEMONSTRATE THE ASSOCIATION BETWEEN WORK-RELATED STRESS AND METABOLIC SYNDROME?}

${ }^{1}$ Nicola Magnavita* ${ }^{1}$ Ilaria Capitanelli, ${ }^{1}$ Erika Alessandra Lops, ${ }^{1}$ Sara Manetta, 2,3 Sergio Garbarino. 'Institute of Public Health, Università Cattolica del Sacro Cuore, Rome, Italy; ${ }^{2}$ Department of Health Sciences, University of Genoa, Genoa, Italy; ${ }^{3}$ Department of Neuroscience, Rehabilitation, Ophthalmology, Genetics and Maternal/Child Sciences (DINOGMI), University of Genoa, Genoa, Italy

\subsection{6/oemed-2018-ICOHabstracts. 1626}

Introduction Work-related stress (WS) has been proposed as a risk factor for the development of the metabolic syndrome (MetS). The number of studies, however, is limited, and results are conflicting. The occupational health physician can give an effective contribute to clarify this point by systematically collecting data from workers waiting for medical examination.

Methods During 2016, the workers were invited to complete a questionnaire that included the Effort-Reward Imbalance scale (ERI) of Siegrist and the Support scale from the DCS model of Karasek, before their medical examination in the workplace. 1904 persons (male 32.6\%, female 67.4\%) participated in the study. The mean age was 47.35 \pm 9.38 . Health data were obtained from medical surveillance records.

Results The prevalence of workers with high blood pressure (316, 16.6\%), high cholesterol/reduced HDL-cholesterol (511, 26.8\%), high triglycerides (195, 10.2\%), high blood glucose
(91, 4.8\%), and obesity $(677,35.6 \%)$ led to a diagnosis of MetS in 196 workers (10.3\%). In univariate logistic regression analysis, WS was significantly associated with the occurrence of MetS (OR 1.82; 95\% CI: 1.41 to 2.35). ERI was also significantly associated with hypertriglyceridemia (OR 1.64; 95\% CI: 1.27 to 2.13) and with overweight (OR 1.33; 95\% CI: 1.11 to 1.59 ). The association was still significant in multivariate models, after correction for confounders.

Discussion The observed association between WS and MetS deserves particular attention. The root causes of stress in workers must be investigated so as to have information for prevention.

\section{HEALTH AND WELLBEING- WORK-LIFE IMBALANCE IN DEVELOPING COUNTRIES}

Janice Green. JN Bank Limited, Kingston, Jamaica

10.1136/oemed-2018-ICOHabstracts. 1627

Introduction The concept of work-life balance, health and well-being is about behaviour change management in ones physical, social, and mental state to attain a measure of stability. This stability can be achieved through creative and substantial preventative and or corrective actions taken in a collaborative manner with other relevant persons.

Professors Jodi Oakman and Siew Chan in their safety journal on the topic Risk management: Where should we target strategies to reduce work-related musculoskeletal disorder; did a study on Australian companies with high reported instances of work related musculoskeletal disorders (WMSDs). In this study, it was found that there was evidence to support the claim that there is a link between WMSD and psychosocial factors. Psychosocial factors such as stress, work place demand, job security and working hours are said to be predicators of WMSDs. The study also revealed that there is an inverse relationship between work-life balance and psychosocial factors. A low level of work-life balance indicates that there is a high level of stress, workplace demand complicated by work-related musculoskeletal disorders.

It's a general belief that the mean age for employees at the reproductive age is 35 years. It is at this crossroads that most employees are working the hardest at balancing their priorities; work, family, social and their personal development. It is at this juncture of their lives that the psychosocial factors of work related stress, economic stress, physical inactivity and life style illnesses take centre stage. The imbalance occurs as one or two priorities are given more attention and the focus on the others significantly decreases.

From the premise of a proactive employer who is concerned about the health and wellbeing of the workers; behaviour change intervention programs become necessary. These interventions are relevant to ensure that workers remain healthy so that productivity does not suffer and the worker will benefit, eventually with financial rewards. The worker more often is sometimes is not able to individually, and unilaterally, restore the require work-life balance, hence the support of the employer becomes important.

Methods To achieve this balance there has to be a vision of what the behaviour change intervention programme is to achieve. How 